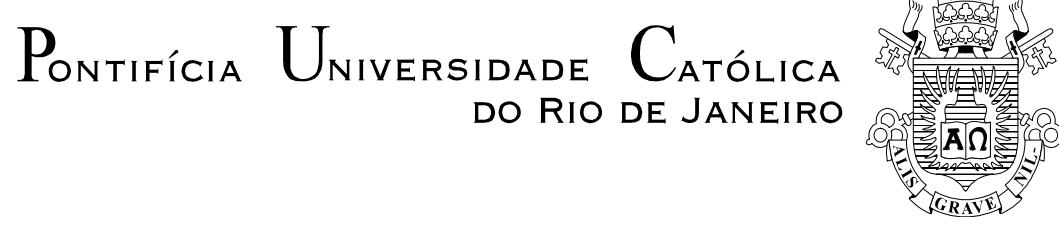

Luís Fernando Ferreira

Simulação Estática e Dinâmica do Controle de Tensão por Geradores

Dissertação de Mestrado

Dissertação apresentada como requisito parcial para obtenção do grau de Mestre pelo Programa de Pósgraduação em Engenharia Elétrica do Departamento de Engenharia Elétrica da PUC-Rio.

Orientador: Prof. Ricardo Bernardo Prada

Rio de Janeiro Julho de 2006 


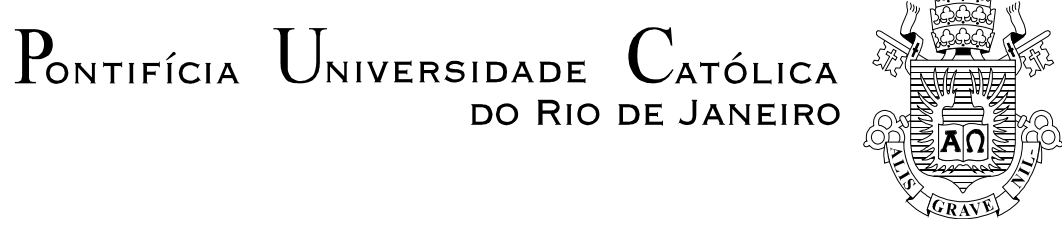

Luís Fernando Ferreira

Simulação Estática e Dinâmica do Controle de Tensão por Geradores

Dissertação apresentada como requisito parcial para obtenção do grau de Mestre pelo Programa de PósGraduação em Engenharia Elétrica do Departamento de Engenharia Elétrica do Centro Técnico Científico da PUCRio. Aprovada pela Comissão Examinadora abaixo assinada.

\author{
Prof. Ricardo Bernardo Prada \\ Orientador \\ Departamento de Engenharia Elétrica - PUC-Rio
}

Prof. Júlio César Rezende Ferraz CEPEL

Prof. Carlos Henrique Costa Guimarães UFF

Prof. José Eugenio Leal Coordenador Setorial do Centro

Técnico Científico

Rio de Janeiro, 13 de julho de 2006 
Todos os direitos reservados. É proibida a reprodução total ou parcial do trabalho sem autorização da universidade, do autor e do orientador.

\section{Luís Fernando Ferreira}

Graduou-se em Engenharia Elétrica na Universidade Federal de São João Del Rei em 2003. Atualmente é consultor na área de energia da ICF International.

Ficha catalográfica

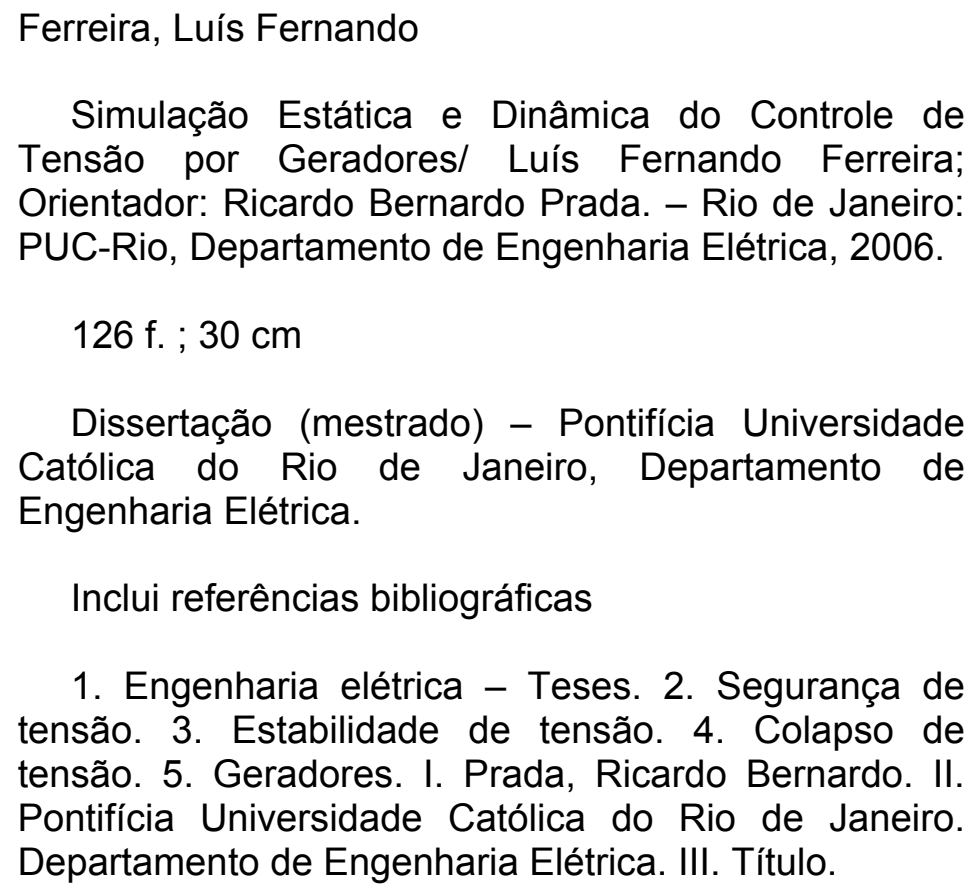

1. Engenharia elétrica - Teses. 2. Segurança de tensão. 3. Estabilidade de tensão. 4. Colapso de tensão. 5. Geradores. I. Prada, Ricardo Bernardo. II. Pontifícia Universidade Católica do Rio de Janeiro. Departamento de Engenharia Elétrica. III. Título.

CDD: 621.3 
À minha querida mãe, Efigênia Ferreira pelo amor, apoio, dedicação e confiança 


\section{Agradecimentos}

A Deus.

A minha mãe Efigênia Ferreira, por seus bons conselhos, infinito amor, apoio econômico e por estar sempre a meu lado, sem ela seria impossível terminar o mestrado.

A meu pai Nelson Ferreira, por me cuidar e me guiar nesta vida, a minha avó Holanda, a minha tia Elizabeth e ao meu avô Geraldo que mesmo sem sua presença física, já me basta às recordações.

Muito especialmente, agradeço ao meu orientador Ricardo Bernardo Prada pelo permanente apoio nas diferentes etapas do desenvolvimento nesta dissertação, por sua atenção e paciência na discussão dos diferentes aspectos relacionados ao tema de pesquisa.

Agradeço também a Luis Cláudio Ferreira (ONS), João Passos Filho e Ricardo Henriques (CEPEL) pelas inestimáveis colaborações para o desenvolvimento da tese.

Ao CNPQ e à PUC-Rio, pelos auxílios concedidos, sem os quais este trabalho não poderia ter sido realizado.

À Bianca pelo amor e amizade. A todos os amigos da ICF e do curso de Pós-Graduação da Engenharia Elétrica, muito especialmente ao amigo Carlos Portugal. 


\section{Resumo}

Ferreira, Luís Fernando; Prada, Ricardo Bernardo (Orientador). Simulação Estática e Dinâmica do Controle de Tensão por Geradores. Rio de Janeiro, 2006. 126p. Dissertação de Mestrado - Departamento de Engenharia Elétrica, Pontifícia Universidade Católica do Rio de Janeiro.

O problema tratado nesta dissertação é a relação oposta entre a tensão de excitação de geradores e compensadores síncronos e a tensão controlada, quando o sistema de transmissão da área encontra-se muito carregado. Neste caso, a capacidade nominal de um gerador / compensador não seria útil para manter a tensão controlada. Devido à relação oposta, uma maior excitação da máquina iria abaixar a tensão controlada. O controle automático iria continuar agindo, abaixando ainda mais a tensão. Este mecanismo pode levar o sistema ao colapso e foi verificado em ponto de operação real do sistema brasileiro. Esse fenômeno ocorre quando a injeção de potência na rede de transmissão ou distribuição é elevada. Com o advento da geração distribuída, co-geração e produtores independentes, usualmente conectados à rede existente em níveis de tensão mais baixas, têm-se observado ocorrências do fenômeno. O objetivo do trabalho é então entender melhor as situações operativas reais que levam à ocorrência do fenômeno, principalmente quando existem vários equipamentos de controle de tensão ao redor do gerador em análise. A abordagem do problema baseou-se na verificação do comportamento do gerador / compensador como dispositivo de controle de tensão, no domínio do tempo e em regime permanente. Avaliaram-se as ações de controle do mesmo a partir de sete tipos de análise distintas para pontos de operação na região normal e anormal da curva SV. A real existência do fenômeno foi comprovada através de algumas destas análises. Porém, conclui-se que nem todas as formas de análise no domínio do tempo fazem uma avaliação completa do fenômeno. Dentre essas, estão a análise dinâmica agregada e a análise dinâmica agregada sob influência dos equipamentos de controle de tensão, que para pontos de operação na região anormal da curva SV não responderam em concordância com os outros tipos de análise.

\section{Palavras-chave}

Estabilidade de tensão, controle de tensão, segurança de tensão, simulação no domínio do tempo e simulação em regime permanente. 


\section{Abstract}

Ferreira, Luís Fernando; Prada, Ricardo Bernardo (Advisor). Static and Dynamic Simulation for Generator Voltage Control. Rio de Janeiro, 2006. 126p. MSc. Dissertation - Departamento de Engenharia Elétrica, Pontifícia Universidade Católica do Rio de Janeiro.

The problem addressed in this research is the opposite relationship between the, synchronous generator / compensator excitation voltage and the controlled voltage when nearby network is heavily loaded. In this situation, the nominal capacity of a generator / compensator would not keep the voltage controlled. Due to the opposite relationship, the higher the excitation voltage the lower is the controlled voltage. So, the automatic control would continue acting lowering the voltage. This mechanism, verified in a real operational point of the Brazilian Electric System, can lead the system to collapse. This phenomenon occurs when the power injection into the network is high. It is prone to occur in the new scenario of distributed generation connected to already existing low voltage networks. The objective of this work is to understand the actual operative situations that lead to the occurrence of the phenomenon, mainly when there are several voltage control devices nearby the generator. The analysis of the problem was based on the verification of the generator behaviour as a voltage control device, in time domain simulation and in steady state. The control actions were evaluated from seven different ways for operating points in the normal and the abnormal region of the SV curve. The actual existence of the phenomenon was proven through some of these analyses. However, some of the time domain simulations did not evaluated the phenomenon completely. Among them, the aggregated dynamic analysis and the aggregated dynamic analysis under influence of other voltage control devices have not got the expected responses for the abnormal region of the SV curve, in comparison with other analysis.

\section{Keywords}

Voltage stability, voltage control, voltage security, time domain simulation, steady state simulation. 


\section{Sumário}

1. Introdução 16

1.1. Considerações Gerais 16

$\begin{array}{ll}\text { 1.2. Objetivo } & 17\end{array}$

$\begin{array}{ll}\text { 1.3. Estrutura da Dissertação } & 17\end{array}$

2. Estabilidade de Tensão 18

2.1. Introdução 18

2.2. Caracterização do Fenômeno da Estabilidade de Tensão: A Máxima Potência Transmitida 18

2.3. Caracterização do Fenômeno de Estabilidade de Tensão: Efeitos Opostos das Ações de Controle de Tensão 27

2.4. Índices de Avaliação das Condições de Segurança de Tensão 30

2.4.1. Módulo do Determinante da Matriz[D’] 31

$\begin{array}{ll}\text { 2.4.1.1. Sistema de Duas Barras } & 32\end{array}$

2.4.1.2. Sistema Multi-Nó $\quad 34$

2.4.2. Sinal do Determinante da Matriz [D'] 36

2.4.3. Margem de Potência 38

2.5. Conceituação do Fenômeno de Relações Opostas Envolvendo a Tensão 39

2.5.1. Potência Reativa Gerada x Tensão Terminal para o Controle de Tensão 39

2.5.2. Tensão Interna x Tensão Terminal para o Controle de Tensão 41

2.6. Conclusões 44

3. Avaliação da Existência do Fenômeno 45

3.1. Descrição e Importância do Problema 45

3.2. Índices de Estabilidade de Tensão 46

3.3. Análise Através de Algoritmo de Fluxo de Carga 48

3.4. Análise da Tensão de Excitação x Tensão Terminal 51

3.5. Análise Dinâmica $\quad 54$

3.5.1. Análise Dinâmica Agregada $\quad 59$

3.6. Simulador Numérico para Análise no Domínio do Tempo 63

3.6.1. Introdução 63

3.6.2. Desenvolvimento 63

$\begin{array}{ll}\text { 3.6.3. Resultados } & 65\end{array}$

3.7. Conclusões 78

4. Análise em Regime Permanente e no Domínio do Tempo de Problemas de Estabilidade de Tensão em Sistemas Reais 81

4.1. Introdução $\quad 81$

4.2. Índices de Estabilidade de Tensão $\quad 81$

4.3. Análise Através de Algoritmo de Fluxo de Carga 83

4.4. Análise da Tensão de Excitação x Tensão Terminal 85

4.5. Análise Dinâmica $\quad 86$

4.5.1. Análise Dinâmica Agregada 92

4.5.2. Análise Dinâmica Agregada sob Influência dos Equipamentos de Controle de Tensão 96

4.5.3. Comportamento dos Equipamentos de Controle de Tensão 99 
4.5.3.1. Funcionamento dos LTCs 99

4.5.3.2. Funcionamento do Compensador Síncrono 104

4.5.3.3. Funcionamento do Compensador Estático 106

$\begin{array}{ll}\text { 4.6. Conclusões } & 107\end{array}$

$\begin{array}{ll}\text { 5. Conclusões } & 110\end{array}$

6. Referências Bibliográficas 113

Apêndice A - Metodologia Utilizada para a Construção da Ferramenta de Simulação Dinâmica 


\section{Lista de Tabelas}

Tabela 3.1 - Ponto de Operação na Região Normal da Curva SV 47

Tabela 3.2 - Ponto de Operação na Região Anormal da Curva SV

Tabela 3.3 - Índices de Estabilidade de Tensão na Região Normal da Curva SV

Tabela 3.4 - Índices de Estabilidade de Tensão na Região Anormal da Curva SV

Tabela 3.5 - Análise Através de Algoritmo de Fluxo de Carga na Região Normal de Operação

Tabela 3.6 - Análise Através de Algoritmo de Fluxo de Carga na Região Anormal de Operação

Tabela 3.7 - Tensão de Excitação e Tensão Terminal a partir de um Ponto na Região Normal de Operação

Tabela 3.8 - Tensão de Excitação e Tensão Terminal a partir de um Ponto na Região Anormal de Operação

Tabela 3.9 - Análise Conclusiva dos Testes Realizados 78

Tabela 3.10- Análise Comparativa dos Testes

Tabela 4.1 - Relatório do EstabTen para a Região Normal da Curva SV

Tabela 4.2 - Relatório do EstabTen para a Região Normal da Curva SV

Tabela 4.3 - Análise Através de Algoritmo de Fluxo de Carga na Região Normal de Operação

Tabela 4.4 - Análise Através de Algoritmo de Fluxo de Carga na Região Anormal de Operação

Tabela 4.5 - Tensão de Excitação e Tensão Terminal a partir de um Ponto na Região Normal de Operação

Tabela 4.6 - Tensão de Excitação e Tensão Terminal a partir de um Ponto na Região Anormal de Operação 


\section{Lista de Figuras}

Figura 2.1 - Sistema-Teste de Duas Barras $\quad 18$

Figura 2.2 - Lugar Geométrico da Tensão na Carga para Todos os Níveis de 24

Potência Ativa Constante e para Alguns Níveis de Potência Reativa Constante

Figura 2.3 - Gráfico QV com o Lugar Geométrico da Tensão na Carga para P 25

Constante,para $Q$ constante, para $\phi$ constante

Figura 2.4 - Curva PV Referente ao Sistema de Duas Barras da Figura $2.1 \quad 27$

Figura 2.1 - Sistema de Duas Barras com Capacitor na Barra de Carga 28

Figura 2.6 - Curvas PV Antes e Depois da Instalação do Capacitor na Barra de Carga

Figura 2.7 - Localização dos Vetores Gradiente de $P_{i}$ e $Q_{i}$ no Plano V $\Theta$

Figura 2.8 - Sinal da Margem na Curva SV 39

Figura 2.9 - Sistema de 3 Barras 41

Figura 3.1 - Sistema de 2 Barras 46

Figura 3.2 - Tensão Terminal x Fluxo de Potência Reativa na Região Normal $\quad 50$

de Operação

Figura 3.3 - Tensão Terminal x Fluxo de Potência Reativa na Região Anormal 51

de Operação

Figura 3.4 - Tensão Interna x Tensão Terminal a partir de Um Ponto na 53

Região Normal de Operação

Figura 3.5 - Tensão Interna x Tensão Terminal a partir de Um Ponto na 54

Região Anormal de Operação

Figura 3.6 - Variação da Tensão na Barra Interna no Tempo 56

Figura 3.7 - Variação da Tensão Terminal do Gerador no Tempo 57

Figura 3.8 - Variação do Fluxo de Potência Reativa que Chega na Barra 57

Terminal no Tempo

Figura 3.9 - Variação da Tensão Interna no Tempo 58

Figura 3.10 - Tensão Terminal 58

Figura 3.11 - Variação do Fluxo de Potência Reativa que Chega na Barra 59

Terminal no Tempo

Figura 3.12 - Tensão de Excitação $\quad 60$

Figura 3.13 - Tensão Terminal $\quad 60$

Figura 3.14 - Fluxo de Potência Reativa Chegando na Barra Terminal 61

$\begin{array}{ll}\text { Figura } 3.15 \text { - Tensão de Excitação da Máquina } & 61\end{array}$

Figura 3.16 - Tensão Terminal 62

Figura 3.17 - Fluxo de Potência Reativa Chegando na Barra Terminal 62

Figura 3.18 - Fluxo de Potência Reativa Chegando na Barra Terminal 66

Figura 3.19 - Fluxo de Potência Reativa Chegando na Barra Terminal 66

$\begin{array}{ll}\text { Figura } 3.20 \text { - Tensão Terminal } & 67\end{array}$

Figura 3.21 - Tensão Terminal $\quad 67$

$\begin{array}{ll}\text { Figura } 3.22 \text { - Tensão de Excitação da Máquina } & 67\end{array}$

Figura 3.23 - Tensão de Excitação da Máquina 68

Figura 3.24 - Tensão de Excitação da Máquina 69

$\begin{array}{ll}\text { Figura } 3.25 \text { - Tensão de Excitação da Máquina } & 69\end{array}$ 
Figura 3.26 - Tensão Terminal $\quad 70$

Figura 3.27 - Tensão Terminal $\quad 70$

Figura 3.28 - Fluxo de Potência Reativa Chegando na Barra Terminal 71

Figura 3.29 - Fluxo de Potência Reativa Chegando na Barra Terminal 71

Figura 3.30 - Tensão de Excitação da Máquina $\quad 72$

Figura 3.31 - Tensão de Excitação da Máquina 72

Figura 3.32 - Tensão Terminal

Figura 3.33 - Tensão Terminal $\quad 73$

Figura 3.34 - Fluxo de Potência Reativa Chegando na Barra Terminal 74

Figura 3.35 - Fluxo de Potência Reativa Chegando na Barra Terminal 74

Figura 3.36 - Tensão de Excitação da Máquina $\quad 75$

Figura 3.37 - Tensão de Excitação da Máquina $\quad 75$

$\begin{array}{ll}\text { Figura } 3.38 \text { - Tensão Terminal } & 76\end{array}$

Figura 3.39 - Tensão Terminal $\quad 76$

Figura 3.40 - Fluxo de Potência Reativa Chegando na Barra Terminal 77

Figura 3.41 - Fluxo de Potência Reativa Chegando na Barra Terminal $\quad 77$

Figura 4.1 - Tensão Terminal x Fluxo de Potência Reativa na Região Normal 84

de Operação

Figura 4.2 - Tensão Terminal x Fluxo de Potência Reativa na Região Anormal 85

de Operação

Figura 4.3 - Diagrama Unifilar sem a Representação da Barra Interna $3978 \quad 86$

Figura 4.4 - Diagrama Unifilar com a Representação da Barra Interna $3978 \quad 86$

Figura 4.5 - Tensão Interna x Tensão Terminal a partir de um Ponto na Região 88

Normal de Operação

Figura 4.6 - Tensão Interna x Tensão Terminal a partir de um Ponto na Região 88

Anormal de Operação

Figura 4.7 - Tensão de Excitação da Máquina $\quad 90$

Figura 4.8 - Tensão Terminal 90

Figura 4.9 - Fluxo de Potência Reativa Chegando na Barra Terminal 90

Figura 4.10 - Tensão de Excitação da Máquina 91

Figura 4.11 - Tensão Terminal $\quad 91$

Figura 4.12 - Fluxo de Potência Reativa Chegando na Barra Terminal 92

Figura 4.13 - Tensão de Excitação da Máquina 93

Figura 4.14 - Tensão Terminal 93

Figura 4.15 - Fluxo de Potência Reativa Chegando na Barra Terminal 94

Figura 4.16 - Tensão de Excitação da Máquina 94

Figura 4.17 - Tensão Terminal 95

Figura 4.18 - Fluxo de Potência Reativa Chegando na Barra Terminal 95

Figura 4.19 - Região de Análise 96

Figura 4.20 - Tensão de Excitação da Máquina 98

Figura 4.21 - Tensão na Barra Terminal $3977 \quad 98$

Figura 4.22 - Fluxo de Potência Reativa na Barra Terminal $3977 \quad 99$

Figura 4.23 - Tensão na Barra 175 de Campos 100

Figura 4.24 - Variação do Tap na Barra 175 de Campos 100

Figura 4.25 - Variação da Tensão na Barra 177 de Vitória 101

Figura 4.26 - Variação do Tap na Barra 177 de Vitória 101

Figura 4.27 - Tensão na Barra 3966 de Macaé Merchant 102

Figura 4.28 - Tensão na Barra 140 de Adrianópolis 102

Figura 4.29 - Tensão na Barra 147 de Campos 103

Figura 4.30 - Tensão na Barra 149 de Vitória 103

Figura 4.31 - Tensão na Barra 45 de Vitória 105 
Figura 4.32 - Tensão de Excitação da Máquina

Figura 4.33 - Potência Reativa na Barra 45 de Vitória

Figura 4.34 -Tensão na Barra 46 de Campos

106

Figura 4.35 - Potência Reativa na Barra 46 de Campos 


\section{Abreviaturas e Siglas}

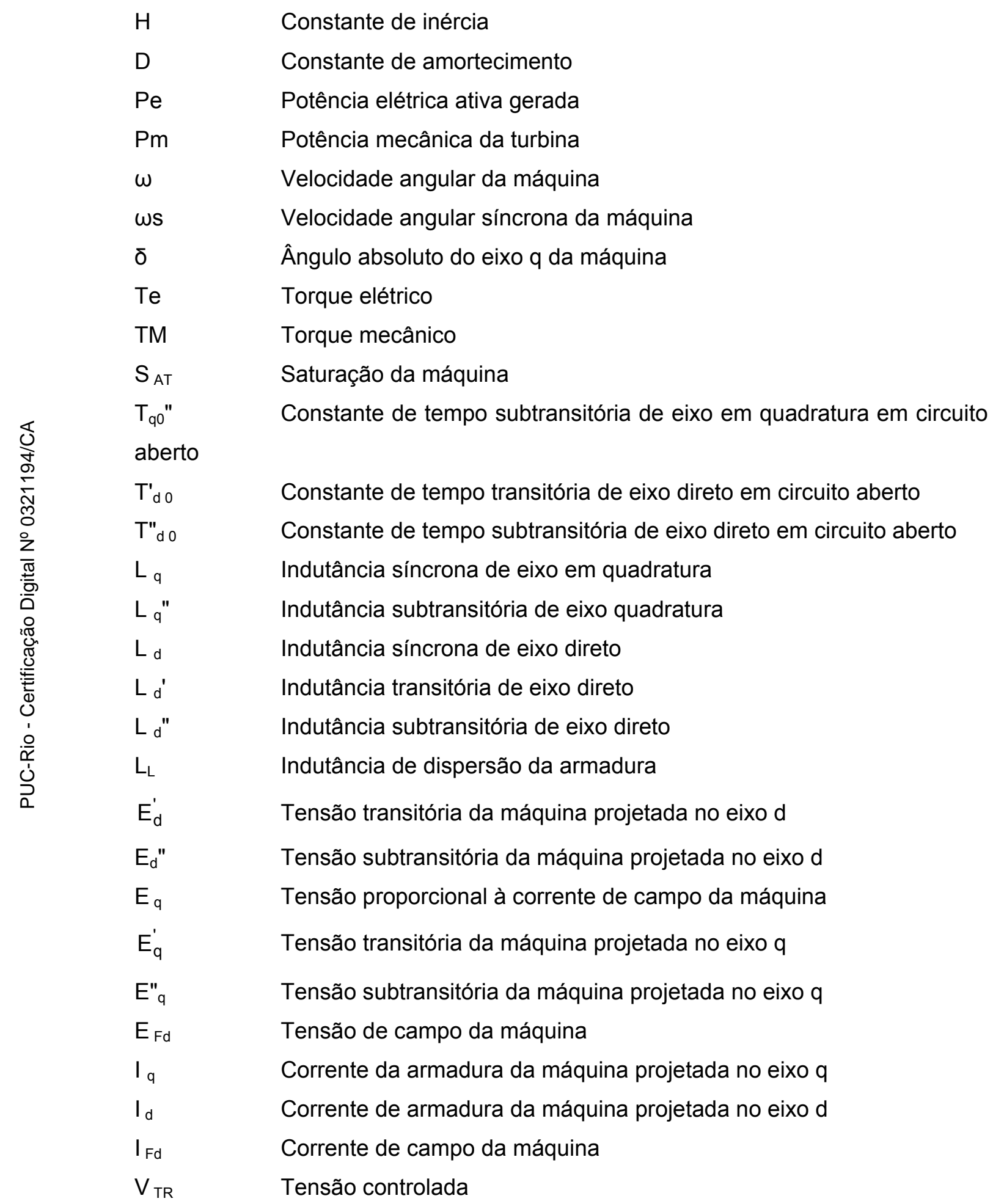


$V_{\text {SAD }} \quad$ Sinal estabilizador aplicado no regulador de tensão

$V_{\text {Ref }} \quad$ Sinal de referência

$\mathrm{T}_{\mathrm{m}} \quad$ Constante de tempo do transdutor de tensão

$\mathrm{K}_{\mathrm{a}} \quad$ Ganho do regulador de tensão

$\mathrm{T}_{1} \quad$ Constante de tempo de avanço do compensador de fase do regulador de tensão

$\mathrm{T}_{2} \quad$ Constante de tempo de atraso do compensador de fase do regulador de tensão

$\mathrm{T}_{3} \quad$ Constante de tempo de avanço do compensador de fase do regulador de tensão

$\mathrm{T}_{4} \quad$ Constante de tempo de atraso do compensador de fase do regulador de tensão

$L_{\text {MIN }} \quad$ Limite inferior da tensão de saída do regulador de tensão

$L_{\text {MAX }} \quad$ Limite superior da tensão de saída do regulador de tensão

$R_{L}$

Resistência da linha

$\mathrm{X}_{\mathrm{L}}$

Reatância da linha

LET Limite de estabilidade de tensão

$Z_{t} \quad$ Impedância da linha de transmissão

$\alpha_{t}$

Ângulo da impedância da linha de transmissão

$\mathrm{Z}_{\mathrm{c}} \quad$ Impedância de carga

$\mathrm{P}_{\mathrm{G}}$

Potência Ativa Gerada MW

$\mathrm{Q}_{\mathrm{G}}$

Potência Reativa Gerada Mvar

$\theta_{\mathrm{i}}$

Ângulo da Tensão na Barra i

$\mathrm{V}_{\mathrm{i}}$

Módulo da Tensão na Barra i 